\title{
Server Support for Cooperative Hypermedia Systems
}

\author{
Helge Schütt, Jörg M. Haake \\ GMD-IPSI \\ Postfach 104326 \\ W-6100 Darmstadt \\ Federal Republic of Germany \\ e-mail: \{ schuett, haake\}@ darmstadt.gmd.de
}

\begin{abstract}
While current hypertext technology usually is restricted to single user systems, it is obvious that actual work environments require multi-user systems which support cooperating coworkers. However, previous research prototypes have concentrated either on the support for group work or on appropriate datamodels and storage policies for hypermedia objects. In this paper we discuss the CHS hypermedia engine which combines both approaches: It not only provides shared access to hypermedia objects but also supports cooperating clients in flexible ways.
\end{abstract}

Keywords: Cooperative Hypermedia Systems, Databases, Computer-Supported Cooperative Work

\section{$1 \quad$ Introduction}

In today's workplace there is virtually no task which is solved by an individual alone. Instead, it is teams of people who work together to solve a case, to prepare a document, or to complete a design. If hypermedia systems (cf. [Bus45, Ne165, Con87, Nie90, Kuh91]) want to enter the real world, it is therefore a prime requirement that they support multiple users who work concurrently on the same set of data (cf. [MPS91, LØS92]). Their work has to be synchronized, and they must be allowed to employ different cooperation models. From an architectural standpoint, it is clear that it is desirable to provide a (distributed) server for that purpose. Such a server would

- provide a shared database where hypermedia objects can be stored persistently,

- handle transactions, i.e. schedule concurrency control, perform recovery, and ensure data consistency during multi-user updates to the database,

- provide persistent and non-persistent activity markers on selected data,

- notify cooperating clients when data is updated so that they share the same view of the database.

In the German National Research Center for Computer Science - Integrated Publication and Information Systems Institute (GMD-IPSI), the CHS Cooperative Hypermedia Server has been designed and implemented to provide these services. While previous efforts on the HyperBase hypermedia engine have concentrated on the first two requirements and have been discussed elsewhere (cf. [SS90, Sch92]), CHS also provides the latter two services.

The paper is organized as follows: First we give an overview of the problem and related work. In the main section of this paper we explain CHS's datamodel and describe its system architecture. We then describe the hypermedia authoring environment SEPIA which is the largest application of CHS to date. Finally, we summarize our results and discuss some extensions to CHS.

\section{Cooperative Hypermedia Servers}

First, we give an overview of how working groups of people cooperate with the help of computers and draw some requirements from this discussion. We then review other research activities in the area of cooperative hypermedia systems and server support for hypermedia systems. 


\subsection{Cooperation Models}

When a group of collaborating people works on a task (see [EGR91, Rod91] for an overview of CSCW systems), its members need to access a shared information base (e.g., a document base for co-authoring, or a pool of software modules and documentation for joint software development). Different situations of collaboration arise during the group work process: Sometimes coworkers work on separate parts of the information base (in parallel or at different times), or the way in which documents are processed ensures that at most one person at a time works on a document. We call such a situation an individual work situation. At other times several co-workers may concurrently access some part of the information base but still wish to work as independently as possible. In this loosely-coupled work situation awareness of co-workers' presence and activities is necessary to detect possible conflicts and coordination needs. If several co-workers wish to work in a synchronized way, they engage in tightly-coupled work where they cooperate and coordinate their work in synchronous conference-like "meetings". Here they should be provided with direct communication channels and a shared environment including a common view of the information base (the WYSIWIS principle: "What You See Is What I See").

Actual collaboration proceeds by shifting between these three collaboration modes. The transitions are often prompted by needs for coordination which arise from individual work or through observing activities of co-workers in loosely-coupled work. Therefore, smooth transitions between the modes must be supported.

A groupware application supporting the above collaboration modes requires access to a shared information base. Transactions are needed to synchronize concurrent access and to ensure a consistent state of the information base. To implement synchronization strategies among different sites of a groupware application, activity markers are needed to signal the current status of an object (e.g. is being edited, is checked out, etc.). Update notifications are needed to inform all concurrent sites of new states of the information base to ensure a common view of the information base. This applies not only to data objects but (in tightly coupled mode) also to display information (e.g., position on the screen and display style of information objects).

Furthermore, to support the tightly-coupled mode one needs shared views (WYSIWIS), which can be implemented by communication protocols among cooperating groupware sites. Additional communication facilities, like shared drawing tools, digital audio connections and video networks between distributed workstations, can be used to support informal communication.

To support smooth transitions between the three modes, the shared information base should include additional information which describes the status of the collaboration (e.g., who uses which information, which subgroups work in loosely or tightly-coupled mode). This kind of information can then be used to trigger state transitions among concurrent applications when changes occur. For example, a new co-worker may join a loosely or tightly-coupled session which is already in progress, or someone enters a part of the information base currently used by another co-worker working in individual work mode. In the latter case both applications should shift into looselycoupled mode.

\section{$2.2 \quad$ Related Work}

Three prominent hypertext systems which were designed for group use are Neptune [DS86], the Knowledge Management System (KMS, [AMY88]) and Intermedia [HKR+92]. While the latter two are not based on a client/server architecture, Neptune is based on the Hypertext Abstract Machine (HAM, [CG88]). However, neither of these systems supports different users who want to be informed about updates done by other users. Only by inspecting an object, users can determine whether a change was made, and users cannot be made aware of other users who work "in the neighborhood" (as expressed by the link structure etc.) of the objects they currently work on.

Aquanet [MHRJ91] is a system which supports multiple authors in structuring a knowledge base. It uses a database server to store all relevant information, but apparently it does not exploit its 
database functionality. For example, although Aquanet maintains locks on objects, it does not provide persistent locks which would allow users to lock objects across database sessions. Similarly, Aquanet does not support transactions on the client side. Instead of synchronized group work, Aquanet only supports "semi-synchronous" group work: Clients may poll a "change log" to find out which data has been changed. Although Aquanet broadcasts display information among its clients, this broadcast seems to rely exclusively on the $\mathrm{X}$ window server. Aquanet does not have the notion of a display object where clients may decide whether they want to have their display objects synchronized or not.

In more recent work, several research groups are now engaged in the development of hypermedia engines. The HB1 hyperbase management system [SLH91] and the Distributed Graph Server (DGS, [SSS92]) aim at supporting cooperating hypermedia authors. Both systems are designed as servers which provide shared databases of hypermedia objects to collaborating users. However, neither system has a notion of a transaction (i.e. they do not allow to cluster several basic operations into one atomic operation which is made visible to the global database only when all basic operations have been executed successfully), and neither system supports the notification of collaborating users about changes to the database.

The most advanced system which concentrates on these group work aspects is the EHTS system [Wii91]. This system is based on a hypermedia engine HyperBase (which was named independently from the one we have developed at GMD-IPSI). This HyperBase is a server which not only maintains a database of shared hypermedia objects but also allows clients to subscribe to events and to lock objects. Clients can thus decide whether they want to be notified about changes to the global database. However, all this collaboration information is not stored persistently but only stored with the server process. Therefore, clients cannot recover when that process goes down. Furthermore, EHTS does not support transactions and does not provide persistent locks.

\section{CHS, a Cooperative Hypermedia Server}

To support collaborating authors of hypermedia documents, we have designed the CHS cooperative hypermedia server. Previous research concentrated on a datamodel for hypermedia objects, the sharing of objects, appropriate transaction schemata, balancing the load between clients and the server, and caching strategies for the clients, and has been reported elsewhere [SS90, Sch92]. This research has resulted in the implementation of the HyperBase hypermedia engine. In this section we first explain the data model of CHS and the enhancements to HyperBase's architecture which are necessary to allow synchronized, collaborating client applications.

\subsection{The Datamodel of CHS}

The datamodel of CHS consists of hypermedia objects (nodes, links, and composite objects), and collaboration information (users and activity markers). Each of these concepts is described below. Hypermedia objects are discussed in detail in [SS90] so that we only briefly review them.

Hypermedia object is an abstract class which defines the basic properties of all hypermedia objects. These include the following four features:

- Each hypermedia object is uniquely identified through an object identifier (an OID).

- Each hypermedia object can be linked to any other hypermedia object.

- Each hypermedia object can be a subobject of any number of composite objects.

- Each hypermedia object can carry an arbitrary number of user-defined attributes which can be added and deleted at run time.

Nodes are hypermedia objects which carry a content. This content is of some basic type (string, byte array, etc.) and is not interpreted by the server. Thus nodes are the basic hypermedia objects and roughly correspond to cards in HyperCard [Sha91] or frames in KMS [AMY88]. 
Links are directed references between two hypermedia objects (including other links). Although links are directed, CHS offers functions to retrieve both the links which start and those which end in any given object, and it can also retrieve the objects which are the source or destination of these links.

Composite objects are partially ordered collections of other hypermedia objects. For each composite object, CHS offers functions which retrieve the immediate subobjects or recursively all subobjects of that object. In addition to this, for each hypermedia object $\mathrm{CHS}$ provides functions which retrieve the composite objects it is a subobject of, or recursively all composite objects it is an indirect subobject of.

CHS supports the creation, deletion, and update of these objects. In addition to this, CHS allows to navigate along chains of linked objects or through clusters represented by composite objects (hypertext functionality). CHS also provides closed nested transactions which allow a client to group operations into one atomic operation. For example in SEPIA (see below) each object that is visible at the user interface corresponds to two objects in the internal representation. One object is the hypermedia object the user is conceptually aware of, the other is a visual container object that carries all display information (location on the screen, color, etc.) for that object. Here every create- and delete-operation uses transactions to ensure that no incomplete objects are created in the database.

CHS maintains collaboration information to enable clients to exchange information. On the one hand this means that CHS must keep a database of current users. On the other hand, clients must be able to protect objects so that no other client modifies them during a given time span.

In CHS, a user record consists of the name of the user, the machine he works on, and a port through which his client is willing to accept external communication requests. Through that port it opens a socket communication channel to exchange data with other clients. Each such user record can be identified through a user-id (UID) which is unique for the given database. Using UIDs ensures that even if the same user runs two client applications on the same machine, these clients can still be distinguished.

The port number of a user record can either be a valid port or a distinguished null value HBNOPORT. CHS does not require that clients be able to talk to each other, but if they choose to, they can do it. A user record is automatically created by CHS when a client logs into a database. The only information a client has to provide is its port number; everything else is determined by CHS. As additional functions (compared to HyperBase), CHS allows clients to read a specific user record or all user records.

Cooperating users must inform each other about the objects they (intend to) modify in the global database. To do this, CHS offers activity markers which can be placed on hypermedia objects. Each hypermedia object may carry an arbitrary number of activity markers, where each activity marker consists of an owner (given by his UID) and a severity value. Activity markers can be persistent or non-persistent. The distinction between the two is that markers of the first category survive across sessions while markers of the second category are released when a client logs out from the database.

Wiil's HyperBase offers locks both on whole objects and on single attributes [Wii91]. Wiil has observed that these locks should not be interpreted as strict locks in the sense of database locks because this would inhibit the desired cooperation behavior. Therefore, he allows clients to read locked objects so that a user can navigate through a hyperdocument even while it is being edited.

In CHS we carry this approach further and do not constrain the way in which clients react to activity markers. As an example, consider a node which carries a HBWRITEMARKER. As one policy, clients may decide that this object is invisible to other clients, similar to a write lock in conventional databases. As another policy, other clients might still be allowed to read the marked data, for example to copy the object and derive a new version of it. Still going further, an applica- 
tion may even allow other clients to read and write the marked object, for example when that object is subject to shared editing. Here the marker affects only those users who do not participate in the shared editing session.

Clients can create a marker both unconstrained and in an atomic test-and-set operation. They can read markers of everybody and delete the markers they own both individually and clustered by severity value. When clients log out from a database, $\mathrm{CHS}$ ensures that all non-persistent markers of that client are removed. If a user does not own any persistent markers, his user record is also removed from the database. Otherwise, the user record is only marked as HBNOTLOGGEDIN and is deleted when the user resumes his session. Resuming a session means that all markers which are owned by the old UID are now transferred to the new UID.

\subsection{Architecture}

To fulfill the requirements discussed in the previous sections, we propose the following system architecture (cf. figure 1). The user interface is handled by client workstations. Each workstation maintains a set of tools through which a user interacts with the hypertext objects. These clients use a shared hyperdocument database to persistently store their objects. This database also maintains the activity markers which prevent users from interfering with each other, and it handles concurrency control on critical regions. In addition to this, clients notify each other about changes which occur. These notifications are distributed by a central broadcast server process which ensures that updates are transmitted safely and sequentially. In the remainder of this section we discuss how these components interact.

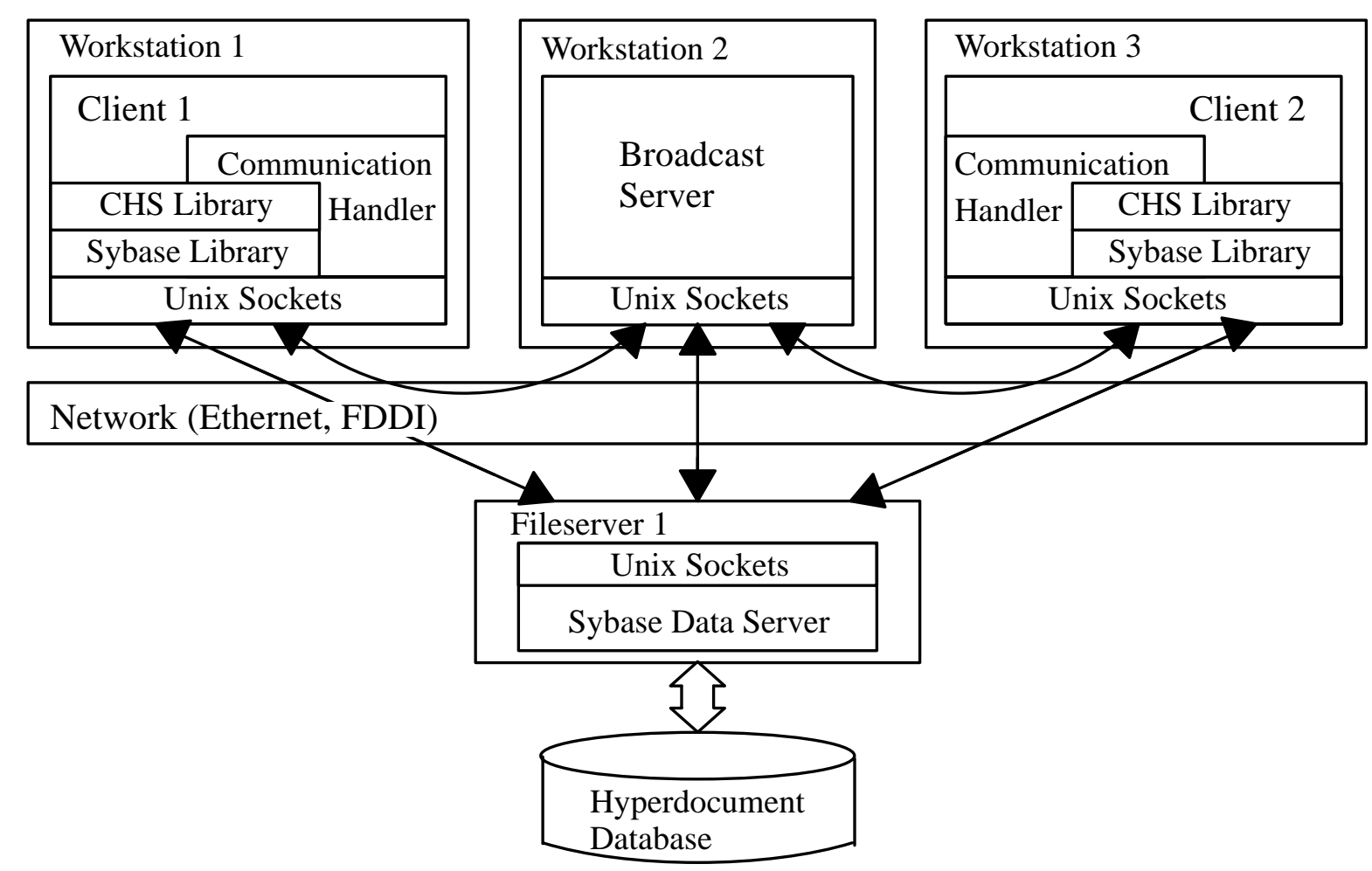

Figure 1: System Architecture

When a user wants to run an application, the following actions happen: First, he logs into the system and specifies a database he wants to work on. CHS then records him in the database together with his workstation and the port through which the client process can be reached. The client then searches the database for a broadcast server which is registered in the database and is willing to accept broadcast requests. If no such broadcast server is found, the client asks to start a new one. An atomic test-and-set operation offered by CHS ensures that only one such request is acknowl- 
edged so that at most one broadcast server exists for each database. Now the client connects itself to the broadcast server, which in turn informs the other registered clients about the new user. These can then retrieve the new user information from the database.

Note that with this approach the client process does not need to know any information about the broadcast server or other client processes in advance. It only specifies the database it wants to work on, and all other information about the broadcast server and the other clients (machines they run or, port numbers, etc.) is maintained by CHS.

As all information (including collaboration information) is stored persistently in the database, this architecture survives crashes of the broadcast server or of any client process. (This is one main advantage over systems like the HyperBase of Aalborg University [Wii91] where collaboration information is stored with the communication process and is lost when that process dies.) With our approach, any number of clients can restart a broadcast server at any time. CHS offers an atomic test-and-set operation to find out whether a second broadcast server process exists, and a broadcast server will shut itself down if this is the case. A restarted broadcast server then can access the database to retrieve the information it needs to communicate with the client processes.

Similarly, the broadcast server notices when a client can no longer be reached, and it can then close its corresponding socket and remove the client's user record from the database. As a side effect, this also releases all activity markers of that client. The data server also recognizes a client which crashes. It then rolls back all transactions which were not committed by the client and ensures that the global database stays consistent. The only situation which cannot be recovered is a failure of the database server process. But in this case a client can no longer access the data it needs, and it should go down gracefully anyway.

The user now wants to change a piece of data on his client. Data here can be the contents of a node, the structure of the hyperdocument, or the way in which objects and browsers appear on the screen. In order to do this, he first selects the objects that are affected by the change. This leads to selection requests that are issued to the database. If no other user currently edits the objects, these requests are acknowledged and activity markers are attached to these objects. The client receives the acknowledgement and informs the user that the selection is successful, e.g. by changing the color of the selected objects.

The user now edits the objects. This can be done through an appropriate editor or by changing their display information e.g. by moving them around. On completion of the editing process, the changed data is transmitted to the database and the broadcast server is informed that a change has happened. The broadcast server then informs all clients that a change has occurred. The clients then decide whether they need to know about the changed data and, if necessary, access the database to retrieve the changed pieces of data. Note that usually this does not involve any disk access: The data has just been written by a client and is still in main memory of the database server.

Note that the completion of the edit operation does not imply that the activity marker on the objects is also released. From CHS's perspective, markers do not prevent other clients from reading or writing the data. It is the clients' decision which cooperation policy they choose. For SEPIA (see below), we decided that other clients still can read data from a marked object but cannot edit it. Furthermore note that markers can be removed independently from other operations. There is no automatic release of markers after a move / edit operation has been completed. Clients may chose an appropriate policy here as well.

Note also that clients can employ different caching strategies. For some applications it may be desirable to load a snapshot of the hyperdocument database to the client process and not care about changes that occur later. Or, as is done in SEPIA, a client application can have the policy that all cached data is updated at the client side as soon as possible so that a client always displays the latest available state of the database. As frequent updates are costly, such an application would load as little data to the client side as needed to keep the number of forced updates at a minimum. 
In our current implementation, all workstations are SUN SPARC-2 workstations running SUN OS 4.1.2 UNIX. The CHS library is written in C and uses the Sybase DB-library [Syb90] as a database. The broadcast server is also written in $\mathrm{C}$ and uses the standard UNIX socket communication. The networks we use are both a LAN based on an Ethernet and a MAN based on FDDI.

\section{The SEPIA Cooperative Hypermedia Authoring Environment}

A major application of CHS is the SEPIA cooperative hypermedia authoring environment. In this section first we briefly introduce the concepts of SEPIA. After that, we explain SEPIA's cooperation model and describe its implementation using the concepts presented in the previous section.

\subsection{SEPIA at a Glance}

SEPIA is a cooperative hypermedia authoring environment. Its purpose is to support groups of authors who collaboratively create hyperdocuments [HW92]. For the purpose of this paper, we will only explain SEPIA's data model and its cooperation model, but not its cognitive foundation [SHT89] or the construction kit which is available to authors [THH91]. Neither will we discuss the external communication lines (audio- and video links) which allow users to talk to each other while collaboratively working on a hyperdocument. A full description of SEPIA has been published in $[\mathrm{SHH}+92]$.

In SEPIA, the largest unit of data is a project. Such a project roughly corresponds to a book together with all background material in traditional publishing. Each project consists of four activity spaces. These are dedicated workspaces which serve different purposes.

Associated with each activity space is a dedicated browser which offers a certain set of functions on the objects it presents. These objects are hypertext objects, i.e., nodes, links, and composite objects. In SEPIA, nodes are typed, and each activity space provides specific types for nodes. Similarly, links are first-class objects, and each activity space offers a set of link types which are relevant in its context. Composite objects are used in all activity spaces to cluster information. One can also open a browser on a composite object to display its subobjects. For all browsers, a construction kit offers several basic options on how information can be arranged.

SEPIA clients have been implemented in Smalltalk-80, Release 4. SEPIA's data model and transaction schema has been implemented with the help of the SFK frame system [FR92]. The CHS library has been integrated into SEPIA as user-defined primitives of the Smalltalk system.

\subsection{Cooperation in SEPIA}

As browsers are the interaction facility of SEPIA, we decided that the most natural way of introducing cooperation into the system is to bind it to the use of browsers. In the following section we explain how this is done at the user interface level, and how it is implemented with the help of CHS's facilities described above. For a more detailed discussion of SEPIA's collaboration model see [HW92].

As illustrated in figure 2, users view SEPIA as a distributed system in which activity space browsers are linked together through a communication channel. Through browsers, we have introduced the concept of a neighborhood in the hypertext network: A user Y works in the neighborhood of user X iff Y works on objects in the same browser as X. (Strictly speaking, these are not the same browsers but different browsers which display the same composite object.) $\mathrm{X}$ is informed when $\mathrm{Y}$ enters his neighborhood. The sound of a doorbell is used to make $\mathrm{X}$ aware of $\mathrm{Y}$ entering his browser, and the names of $\mathrm{X}$ and $\mathrm{Y}$ are displayed in a status line of the browser. In most cooperative systems, a user is only informed when someone else tries to access the same node he is currently working on. In SEPIA, he is already made aware of other people working in the neighborhood of that node. 


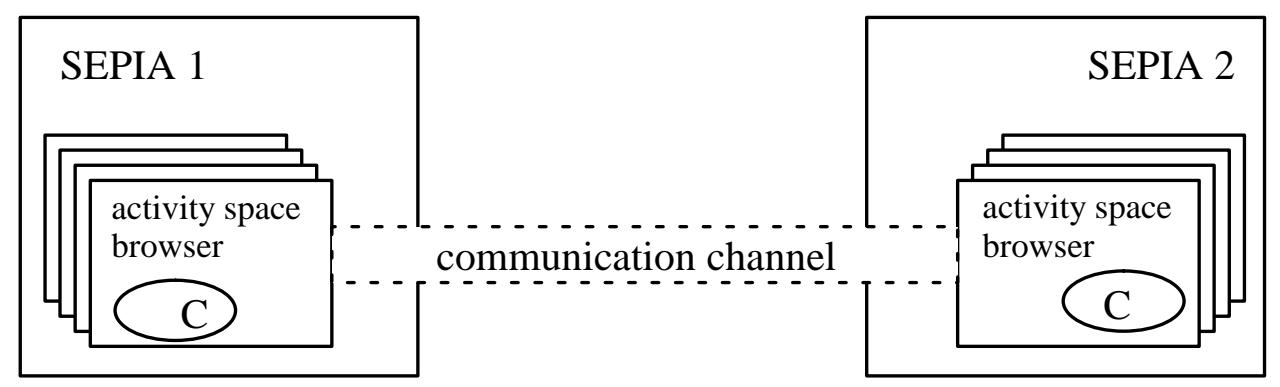

Figure 2: The user's view of cooperating clients

Within each browser, SEPIA uses different colors to represent the activity markers of an object. A white node is not marked by anybody, nodes that have been marked by that client are displayed in yellow, and nodes marked by other clients are displayed in red. Each operation that edits an object (moves it on the screen or actually edits its content) first leads to marking the object before the edit operation can be executed.

Apart from just being aware of others working in his neighborhood, a user may enter a tightly coupled session with a number of collaborating users. In tightly coupled mode, the individual browsers of the users are connected. All resizing of the browser window or scrolling within such a window is immediately transmitted to the other tightly coupled browsers. Here the WYSIWIS (What You See Is What I See) principle is in effect.

From the user's point of view, the architecture presented in the previous section is completely transparent. He only sees his work environment, which is a SEPIA project. Such a project consists of the four activity spaces explained above, each of which is represented on the screen by an activity space browser. Without worrying about the consistency of the shared database or safe communication among the clients, a user can be certain that the changes he makes are not overwritten by others and that the state he sees on his screen is the actual state of the database.

All this is taken care of by CHS. When a client starts, it first opens a socket through which it is willing to exchange information with other clients. The client then logs into a database maintained by CHS. Here it is registered as a user, and the broadcast server informs the other clients about the newcomer. Whenever data is changed, CHS first uses its transactions to ensure that the global database is in a consistent new state before the broadcast server process informs other clients that a change has occurred.

\subsection{Transactions in SEPIA}

CHS provides nested transactions to its clients, and this is one of the features which distinguish CHS from other approaches. In this section, we discuss how this feature is used and what the client has to observe here.

As an example, consider two nodes $\mathrm{N}_{1}$ and $\mathrm{N}_{2}$ which exist in the global database. A user running client $\mathrm{C}_{1}$ now wants to create a link between these nodes. In SEPIA, this translates into the creation of four objects: A link object, a visual container which contains the display information of the link, and two link anchors, one for the source and one for the destination of the link. The global database is in a consistent state only if either none of these four objects exist, or all four of them exist. Therefore, the SEPIA client $\mathrm{C}_{1}$ must ask CHS to ensure that no other client process can read the intermediate states of the database.

Similarly, $\mathrm{C}_{1}$ must have a notion of a local transaction which ensures that on the client side either all four objects or none of these objects exist. To achieve this, a client must keep a log of the objects that have been created or modified during an interaction with a user, and it must be able to rollback the interaction at any point and undo the changes which have been done until this point. 
Note that this log is not subject to multi-user access, i.e. the local transactions need not provide a notion of concurrency control. Note also that the log can be kept in main memory because the global transactions in CHS allow for full recovery. If a client process goes down, its log is lost, but its intermediate state is also lost and has not been committed to the global database. The database realizes that the client went down and rolls back the incomplete transactions.

SEPIA uses a nested commit schema to handle both local and global transactions. $\mathrm{C}_{1}$ first starts a local transaction and a transaction in CHS. It then creates the four objects one after the other. Each of these operations starts subtransactions both on the client side and in CHS. During each operation, $\mathrm{C}_{1}$ checks that the integrity constraints defined among the nodes and links are observed (e.g. that the types of the nodes are legal for the type of link which the user has selected). If such a constraint is not observed, both the local and the global transactions are rolled back. Similarly, if a transaction in CHS fails and is rolled back, the client rolls back its local transactions. When all subtransactions have been committed on the client side, $\mathrm{C}_{1}$ asks $\mathrm{CHS}$ to commit the global transaction. After successful completion of this global transaction, $\mathrm{C}_{1}$ commits its local transaction as well.

Local transactions on the client side are a feature of the SFK frame system [FR92] which has been developed at GMD-IPSI. Note that the transactions on the client side must also allow nesting. However, they have two choices on how they integrate the transaction schema of CHS, and with SEPIA we have done experiments with both of these choices. They may either have each transaction on the client side correspond to one transaction in CHS, or they may collect all changed data on the client side first, and use CHS's transactions only when a user operation has been completed successfully. E.g. a user may choose the "create link ..." option from a menu, select a start node for the link, and then decide to cancel this operation. Here the link, the visual container for the link, and one of the link anchors have already been created and must be removed from the client, and CHS need not be involved at all. The current version of SFK supports this kind of local logging which should decrease communication overhead with the global database maintained by CHS.

As both local and global transactions can be nested to arbitrary depth, clients can cluster any number of operations into new transactions. In our example, SEPIA may introduce a macro operation which creates a node and a link, given an existing node. (There are several examples where this is actually done). Here, SEPIA does not need to redefine the transaction which creates a link (e.g. convert this "main transaction" into a "subtransaction" by shifting the "commit transaction" statement from the "create link"operation to the macro operation), but it merely uses this transaction as a subtransaction of the macro operation without any change to the "begin transaction" / "commit transaction" which has been defined for the "create link" operation.

\subsection{Policies in SEPIA}

As explained in the previous section, a client which uses CHS has to decide on its storage, caching, and update policy with respect to its data objects, and it has to define semantics of the activity markers it uses. In this section we explain SEPIA's choices on these issues.

Our overall goal in the development of SEPIA has been that a user always sees the most recent consistent state of the database. For the storage policy of SEPIA this means that changes are propagated to the database as early as possible. For most updates, this is done as part of the update operation as it appears on the user interface. For continuous updates, however, (e.g. when an object is dragged across the screen) we do not store and broadcast each intermediate step but only propagate the final state of the object. In our example, objects then "jump" across the screens of those users who are only loosely coupled to the user who drags the object. This solution is not completely satisfactory but is reasonable with respect to the speed of current local area networks.

The caching policy of SEPIA says that as few objects as possible are to be loaded from the database. This ensures that very little update communication among the clients is necessary, and data 
is only transferred across the network as it is actually needed. The way this is done in SEPIA is through dummy objects which on the client side take the place of objects which are referenced by other objects but are not yet needed themselves. Such a dummy object replaces itself by an actual object which is retrieved from the database when it is needed by the client.

The update policy of SEPIA is such that every client accepts update requests at any time. It checks whether it has loaded the changed object and loads it from the database if necessary. A client ignores update requests for objects which are not used by it. Each client broadcasts login and logoff requests immediately. Changes to its data objects are broadcast as soon as the corresponding transaction has been completed successfully (i.e. the changed data has been stored with the database). We do not queue or cluster events on the client side; they are sequentialized by the database and the broadcast server.

If a client $\mathrm{C}$ goes down before it can broadcast update notifications, the other clients are in an inconsistent state with respect to the global database. But this is not crucial as transactions ensure that the global database always is in a consistent state and a client which reads an object from the database retrieves this consistent state. In their current status, objects which have been created by $\mathrm{C}$ are not visible to other clients, i.e. they will not wind up in an error. If a client tries to access an object which $\mathrm{C}$ has deleted, CHS will inform that client that this object no longer exists, and the client will remove it from its local cache. If another client modifies an object which already has been modified by $\mathrm{C}$, it overwrites the changes done by $\mathrm{C}$, just as it would if $\mathrm{C}$ were still there.

To support the loosely coupled mode, SEPIA notifies other clients when a new browser is opened or an existing one is closed. As browsers are not hypermedia objects, this cannot not done directly. Instead, each hypermedia object which is displayed by a browser carries an attribute with the list of users who concurrently have opened the object. Thus, instead of broadcasting the changes that are done to a browser, SEPIA broadcasts the changes to this attribute.

SEPIA employs a rather soft locking policy. Each object needs to be marked in order to be changed. An atomic test-and-set operation ensures that only one client has write access to that object. Other clients then recognize the activity marker and start their editors (on nodes) and browsers (on composite objects) in read-only mode. These clients can still read the marked data but not change them.

\section{$5 \quad$ Discussion and Future Work}

In this paper we have described the cooperative hypermedia server CHS (HyperBase, Release 4), which has been designed and implemented at GMD-IPSI. The main features of CHS which support cooperating clients are:

- CHS maintains a shared database of hypermedia objects. The generic datamodel of CHS has proven to be sufficient for hypermedia client applications like SEPIA.

- CHS provides transaction management and both temporary and persistent activity markers. Through these flexible means, client applications can define macro-operations and locking schemata which are appropriate for their corresponding tasks.

- CHS automatically maintains a database of users. Client applications can refer to that database to decide with whom they have to exchange update information, and users can resume sessions in which they had defined persistent activity markers.

The main extension to CHS will be the CoVer context-based version server [Web91] which is being developed at GMD-IPSI. CoVer not only maintains versions of individual objects but also maintains tasks which are the contexts in which these objects are used. It will be integrated into CHS later this year. An application interface then can define a versioning policy which is supported by CHS.

At first glance, it is a good idea to assign access control lists to shared objects, as has been done with the HAM [CG88] and DGS [SSS92]. These lists are additional attributes which restrict ac- 
cess to an object to certain people (i.e. either a single person or a group of collaborating authors). However, Akscyn et al. [AMY88] have reported that such a feature is rarely used in practise, and we therefore do not give it a high priority in our work.

Instead we propose an integration of an organizational database into the server. This database would assign roles to each user of the server and associate access rights with that role. For example, if $X$ is a manager of $Y$ then $X$ has read and write access on all documents written by Y. Furthermore, this database would maintain process structures which describe the way in which documents are created and processed or distributed. For example, if authors $\mathrm{X}$ and $\mathrm{Y}$ want to submit a paper to a conference, the managers of both $\mathrm{X}$ and $\mathrm{Y}$ must be informed about this intended publication, and the paper must not be changed once these managers have released it. In this scenario the datamodel for hypertext objects must be extended such that each object is assigned one or more authors and a role (memo, internal paper, publication, etc.) within the organization.

CHS is currently being reimplemented with the help of the VODAK database management system [KNF+92]. This is a distributed object-oriented database management system which has been developed at GMD-IPSI. With the extended modeling capabilities of VODAK a large part of the application interface (e.g. the mapping of SEPIA objects to CHS objects and the storage policy) can directly be implemented inside the database system. In addition to this, VODAK offers an extended functionality with respect to multimedia data [KNS90] and an improved transaction schema [MRKN92] for better performance.

\section{Acknowledgements}

This paper did greatly benefit from the comments of Norbert Streitz and Erich Neuhold. We wish to thank Boris Bokowski and Christian Schuckmann for their help with the implementation of the clients' interface for SEPIA and their implementation of the broadcast server. And last but not least we wish to thank Lothar Rostek and Dietrich Fischer for their support when we used the SFK and CCK toolkits which proved to be extremely valuable for the development of our system.

\section{References}

[AMY88] R. M. Akscyn, D. L. McCracken, and E. A. Yoder. KMS: A Distributed Hypermedia System for Managing Knowledge in Organizations. CACM, 31(7):820 - 835, July 1988.

[Bus45] V. Bush. As We May Think. Atlantic Monthly, 176(1):101 - 108, June 1945.

[CG88] B. Campbell and J. M. Goodman. HAM: A General Purpose Hypertext Abstract Machine. Communications of the ACM, 31(7):856 - 861, July 1988.

[Con87] J. Conklin. Hypertext: An Introduction and Survey. IEEE Computer, 20(9):17 -41, September 1987.

[DS86] N. M. Delisle and M. D. Schwartz. Neptune: a Hypertext System for CAD Applications. In Carlo Zaniolo, editor, Proceedings of the 1986 ACM-SIGMOD International Conference on Management of Data, pages 132 - 143, Washington, D.C., May 28 - 30, 1986.

[EGR91] C. A. Ellis, S. J. Gibbs, and G. L. Rein. Groupware: Some Issues and Experiences. Communications of the ACM, 34(1):38 - 58, January 1991.

[FR92] D. H. Fischer and L. Rostek. SFK: A Smalltalk Frame Kit - Concepts and Use. Internal working paper, GMD-IPSI, February 17, 1992.

[HKR+92] B. J. Haan, P. Kahn, V. A. Riley, J. H. Coombs, and N. K. Meyrowitz. IRIS Hypermedia Services. Communications of the ACM, 35(1):36 - 51, January 1992.

[HW92] J. M. Haake and B. Wilson. Supporting Collaborative Writing of Hyperdocuments in SEPIA. In Proceedings of the ACM 1992 Conference on Computer Supported Cooperative Work, Toronto, Ontario, November 1-4, 1992.

[KNF+92] W. Klas, E. J. Neuhold, P. Fankhauser, M. Kaul, P. Muth, T. Rakow, and V. Turau. VML The VODAK Model Language, Version 2.0. Working Paper, 1992. 
[KNS90] W. Klas, E. J. Neuhold, and M. Schrefl. Using an Object-Oriented Approach to Model Multimedia Data. Computer Communications 13(4), 204-216, May 1990.

[Kuh91] R. Kuhlen. Hypertext - Ein nicht-lineares Medium zwischen Buch und Wissensbank. Edition SEL-Stiftung. Springer-Verlag, 1991.

[LØS92] D. B. Lange, K. Østerbye, and H. Schütt. Hypermedia Storage. Technical report R 92-2009, Aalborg University, Department of Mathematics and Computer Science, Fredrik Bajers vej 7E, 9220 Aalborg, Denmark, June 1992.

[MHRJ91] C. C. Marshall, F. G. Halasz, R. A. Rogers, and W. C. Janssen Jr. Aquanet: A Hypertext Tool to Hold Your Knowledge in Place. In Proceedings of the 3rd ACM Conference on Hypertext, pages 261 - 275, San Antonio, TX, December 15 - 18, 1991.

[MPS91] K. C. Malcolm, S. E. Poltrock, and D. Schuler. Industrial Strength Hypermedia: Requirements for a Large Engineering Enterprise. In Proceedings of the 3rd ACM Conference on Hypertext, pages 13 - 24, San Antonio, TX, December 15 - 18, 1991.

[MRKN92] P. Muth, T. C. Rakow, W. Klas, and E. J. Neuhold. A Transaction Model for an Open Publication Environment. In A. K. Elmagarmid, editor, Database Transaction Models for Advanced Applications, chapter 6, pages 159 - 218. Morgan Kaufmann, 1992.

[Nel65] T. H. Nelson. A File Structure of the Complex, the Changing, and the Indeterminate. In Proceedings of the 20-th National ACM Conference, pages 84 - 100, Cleveland, OH, August $24-26,1965$.

[Nie90] J. Nielsen. Hypertext and Hypermedia. Academic Press, 1990.

[Rod91] T. Rodden. A survey of CSCW systems. Interacting with Computers, 3(3):319 - 353, December 1991.

[Sch92] H. Schütt. HyperBase: Eine Hypertextmaschine im praktischen Einsatz. In R. Cordes, editor, Proceedings of Hypertext und Hypermedia '92, Munich, September 14 - 16, 1992.

[Sha91] D. Shafer. The Complete Book of HyperCard 2. Addison-Wesley, 1991.

[SHH+92] N. A. Streitz, J. Haake, J. Hannemann, A. Lemke, W. Schuler, H. Schütt, and M. Thüring. SEPIA: A Cooperative Hypermedia Authoring Environment. In Proceedings of the Fourth ACM Conference on Hypertext (ECHT-92), Milan, November 30 - December 4, 1992.

[SHT89] N. A. Streitz, J. Hannemann, and M. Thüring. From Ideas and Arguments to Hyperdocuments: Travelling through Activity Spaces. In Proceedings of the 2nd ACM Conference on Hypertext, pages 343 - 364, Pittsburgh, PA, November 5 - 8, 1989.

[SLH91] J. L. Schnase, J. Leggett, and D. L. Hicks. HB1: Initial Design and Implementation of a Hyperbase Management System. Technical Report TAMU-HRL 91-002, Texas A\&M University, Computer Science Department, Hypertext Research Lab, October 1991.

[SS90] H. Schütt and N. Streitz. HyperBase: A Hypermedia Engine Based on a Relational Database Management System. In A. Rizk, N. Streitz, and J. André, editors, Hypertext: Concepts, Systems, and Applications: Proceedings of the First European Conference on Hypertext, pages 95 - 108, Versailles, France, November 28 - 30, 1990. Cambridge University Press, The Cambridge Series on Electronic Publishing.

[SSS92] D. E. Shackelford, J. B. Smith, and F. D. Smith. A Distributed Graph Storage System for Artifacts in Group Collaborations. Technical Report TR 92-010, The University of North Carolina at Chapel Hill, March 1992.

[Syb90] Sybase, Inc., 6475 Christie Avenue, Emeryville, CA 94608, USA. DB-Library Reference Manual, Release 4.2, May 1990.

[THH91] M. Thüring, J. Haake, and J. Hannemann. What's ELIZA doing in the Chinese Room Incoherent Hyperdocuments and how to Avoid them. In Proceedings of the 3rd ACM Conference on Hypertext, pages 161 - 177, San Antonio, TX, December 15 - 18, 1991.

[Web91] A. Weber. Publishing Tools Need Both: State-Oriented and Task-Oriented Version Support. In Proceedings of the 15th Annual International Computer Software and Applications Conference (COMPSAC-91), pages 633 - 639, Tokyo, Japan, September 11 - 13, 1991.

[Wii91] U. K. Wiil. Using Events as Support for Data Sharing in Collaborative Work. In K. Gorling and C. Sattler, editors, Proceedings of the International Workshop on CSCW, pages $162-$ 177, Berlin, April 9 - 11, 1991. 